\title{
放电位置随机分布的电火花线切割加工仿真模型
}

\author{
白宇飞 王振龙 赵朝夕 王玉魁 \\ (哈尔滨工业大学机电工程学院 哈尔滨 150001)
}

\begin{abstract}
摘要: 在电火花线切割单脉冲放电过程仿真的基础上, 基于有限元软件 ANSYS 提出了一种放电位置随机分布的电火花线切 割加工温度场仿真模型。在仿真过程中, 放电位置随机分布, 并引入 “生死单元技术” 去除高温蚀除的单元, 得到了线切割 连续脉冲放电加工过程的表面效果。然后利用温度场仿真结果计算不同放电参数下的材料去除率, 并基于脉冲利用率和电极 丝换向时间进行修正, 发现修正后材料去除率仿真结果与实际加工结果之间误差小于 $10 \%$, 验证了电火花线切割加工连续脉 冲随机放电的仿真模型的准确性。其中, 电火花线切割加工过程的脉冲利用率、电极丝换向时间是导致仿真结果出现偏差的 主要因素。为了分析实际加工过程的脉冲利用率, 搭建了脉冲利用率检测装置, 对不同伺服条件下的脉冲利用率进行了统计。 关键词: 电火花线切割; 连续脉冲随机放电; 材料去除率; 温度场; 脉冲利用率
\end{abstract}

中图分类号: TG156

\section{Simulation Model of WEDM with Random Distribution of Discharge Position}

\author{
BAI Yufei WANG Zhenlong ZHAO Zhaoxi WANG Yukui \\ (School of Mechatronics Engineering, Harbin Institute of Technology, Harbin 150001)
}

\begin{abstract}
Based on the simulation of single-pulse discharge process of WEDM, a simulation model of temperature field of WEDM with random distribution of discharge position is proposed based on finite element software ANSYS. In the simulation process, the discharge position is randomly distributed, and the "life and death unit technique" is introduced to obtain the erosion effect of the continuous discharge process of the WEDM. Then the temperature field's simulation results are used and the material removal rate under different discharge parameters is calculated. Based on the pulse utilization rate and the wire reversal time, it is found that the error between the modified material removal rate simulation result and the actual machining result is less than $10 \%$, and the accuracy of the continuous pulse random discharge simulation model of WEDM is verified. Among them, the pulse utilization rate and the wire reversal time of the WEDM process are the main factors leading to the deviation of the simulation results. In order to analyze the pulse utilization rate of the actual machining process, a pulse utilization rate's detection device was built to calculate the pulse utilization rate under different servo conditions.
\end{abstract}

Key words: WEDM; continuous pulse random discharge process; material removal rate; temperature field distribution; pulse utilization rate

\section{0 前言}

电火花线切割加工(Wire cut electrical discharge machining, WEDM) 是在电火花加工的基础上于 20 世纪 50 年代末最早在前苏联发展起来的一种工艺 形式, 采用线状电极, 通过极间放电产生大量热, 工件材料在高温下因熔化、气化而被蚀除, 从而实 现对工件的切割 ${ }^{[1]}$ 。但是线切割加一个电场力、磁

20180811 收到初稿, 20190427 收到修改稿
力、热力、流体动力、电化学和胶体化学等综合作 用的复杂过程, 需要设定适当的工艺条件以满足表 面质量、生产效率和成本的要求。目前常用的方法 是针对特定材料和加工条件, 进行大量工艺试验研 究获得最佳加工参数, 试验成本较高。因此建立合 适的有限元仿真模型, 对加工过程进行模拟分析, 对于实际生产具有重要意义。

目前国内外学者多是通过建立单脉冲放电仿真 模型和进行单脉冲放电试验研究电火花加工的蚀除 过程 ${ }^{[2-6]}$, 但是电火花线切割加工是连续脉冲放电的 过程, 加工表面是由无数个放电凹坑叠加而成, 与 
单脉冲放电过程有很大的区别。近年来,一些学者 开始利用有限元法对电火花加工连续脉冲放电进 行研究, 但多采用较为简单的脉冲放电位置排列 方式 ${ }^{[7-9]}$, 而实际脉冲放电过程是一个复杂而且随机 的过程。基于单脉冲放电仿真模型, 本文建立了连 续脉冲随机放电的电火花线切割加工仿真模型, 并 在仿真过程中引入 “生死单元法” 得到了材料不同 加工时刻的蚀除效果, 通过数值计算得到材料去除 率, 通过与实际加工结果比较, 验证了仿真模型的 合理性。该仿真模型可用于新材料的电火花线切割 加工工艺研究, 降低工艺研究过程的实验成本, 以 及分析电火花线切割加工过程的材料蚀除规律。

\section{1 连续脉冲放电过程放电点随机分布}

电火花加工的微观过程可分为四个连续阶段: 极间工作液(介质)电离、击穿, 形成放电通道; 工 作液热分解、电极材料融化、气化热膨胀; 电极材 料抛出; 极间工作液消电离。在极间工作液电离、 击穿, 形成放电通道阶段, 脉冲电压施加于工具电 极和工件形成电场, 当阴极表面某处的电场强度增 大到 $10^{5} \mathrm{~V} / \mathrm{mm}$ 时, 就会有场致电子发射, 形成放 电通道。因此, 在电火花线切割的加工过程中, 放 电点的位置由局部电场强度的分布决定。

在一个放电脉冲加工结束后, 熔化区未被抛出 的电极材料将在放电凹坑表面冷凝, 并在四周形成 稍凸起的翻边, 即熔化凝固层, 如图 1 所示。因此, 当下一个放电脉冲施加在工具电极和工件之间时, 翻边区域由于极间间隙较小、极间电场强度较大, 被击穿形成放电通道的概率较大。但是电极丝和工 件的微观表面是凹凸不平的, 图 2 是考虑电极表面 微观形貌、蚀除凹坑后的极间电场模拟结果, 可以 看出极间实际电场强度是很不均匀的, 同时极间的 电蚀产物也会导致极间局部电场发生畸变, 因此放 电点的位置分布很难实现有序排列。OKADA 等 ${ }^{[10]}$ 利用高速摄像机记录电火花线切割连续脉冲放电加

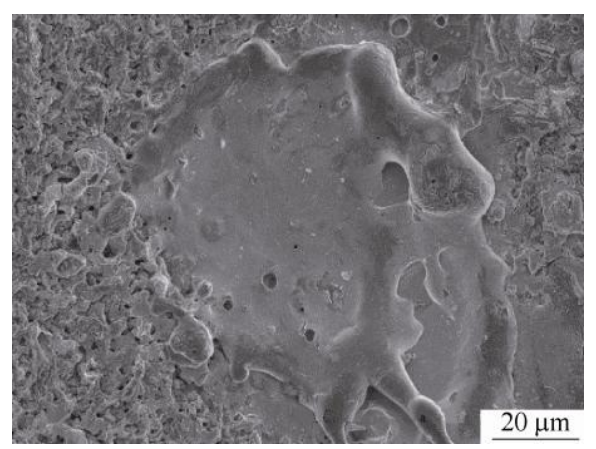

图 1 电火花线切割蚀除凹坑形貌
工过程的放电点位置(图 3), 发现放电点随着时间顺 序的分布排列具有随机性, 证明了放电点位置在加 工区域并非有序排列。为了更加符合电火花线切割 的实际加工过程, 本文认为连续脉冲放电过程的放 电点位置呈随机分布。

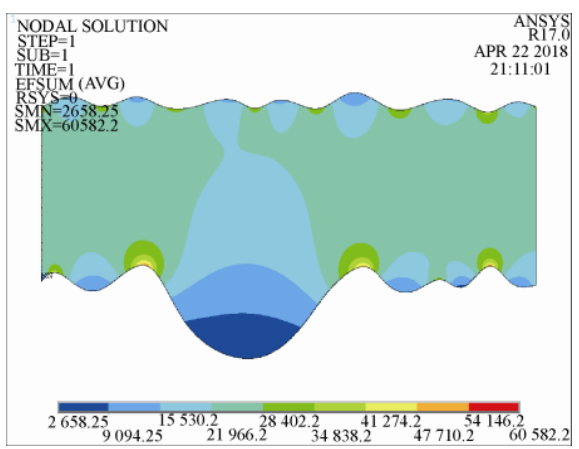

图 2 极间电场强度分布

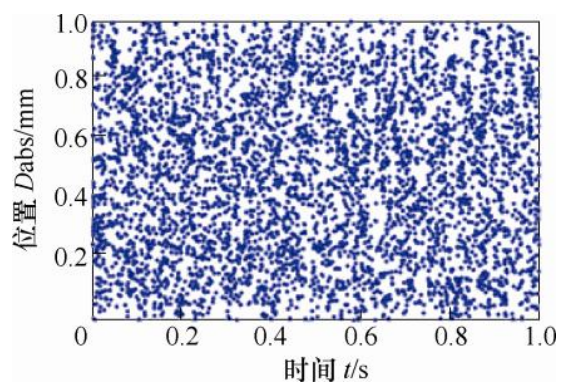

图 3 放电点绝对位置分布

\section{2 连续脉冲放电模型的建立}

\section{1 数学模型的建立}

电火花线切割的温度场分析属于非线性热传导 问题, 根据傅里叶导热定律, 圆柱坐标系下非线性 瞬态三维热传导方程为

$$
\frac{\partial \mathrm{T}}{\partial t}=\frac{\lambda}{c \rho}\left(\frac{\partial^{2} T}{\partial r^{2}}+\frac{1}{r} \frac{\partial T}{\partial r}+\frac{1}{r^{2}} \frac{\partial^{2} T}{\partial \varphi^{2}}+\frac{\partial^{2} T}{\partial z^{2}}\right)+Q(r, \varphi, z, t)
$$

式中, $T$ 为温度; $t$ 为时间; $c 、 \rho$ 和 $\lambda$ 分别为材料的 比热容、密度和导热系数; $Q(r, \varphi, z, t)$ 为热流密度。

连续脉冲放电过程温度场仿真分析需要确定热 流密度、加载面积和持续时间。经分析, 电火花加 工放电通道内的热流密度符合高斯分布, 在某一时 刻, 放电通道内距离放电通道中心为 $r$ 的任一点的 热流密度为

$$
q(r)=\frac{3}{\pi R_{p}^{2}} \eta U I_{p} \exp \left(\frac{-3 r^{2}}{R_{p}^{2}}\right)
$$

式中, $U$ 为极间电压; $I_{p}$ 为峰值电流; $\eta$ 为能量分配 系数; $R_{p}$ 为放电通道半径。

但在电火花线切割加工过程中, 随着电极丝的 
高速移动, 放电通道会发生移动, 因此是近椭圆形。 其中放电通道在脉冲放电时间内沿电极丝运动方向 的移动距离 $d$ 为脉冲宽度 $T_{\mathrm{b}}$ 与电极丝走丝速度 $v$ 乘 积的一半。因此, 放电通道内距离放电通道中心 $(0,0)$ 为 $\left(r_{x}, r_{y}\right)$ 的任意点热流密度表达式为

$$
q(r)=\frac{3}{\pi R_{p}^{2}} \eta U I_{p} \exp \left(-3\left(\frac{r_{x}^{2}}{R_{x}^{2}}+\frac{r_{y}^{2}}{R_{y}^{2}}\right)\right)
$$

式中, $R_{x}=R_{p}, R_{y}=R_{p}+0.5 T_{b} v$ 。

关于极间能量分配系数, 一些学者认为 $\eta$ 不是 固定值, 受脉冲能量大小影响。JOSHI 等 ${ }^{[11]}$ 认为在 不同能量区域有不同的分配系数; SINGH ${ }^{[12]}$ 通过实 验进一步研究了 $\eta$ 与电加工参数之间的关系。本文 基于 Singh 的试验结果, 建立了 $\eta$ 与峰值电流 $I_{\mathrm{p}}$ 和 脉宽 $T_{\mathrm{b}}$ 的数学回归模型

$$
\eta=6.834+0.327 \times I_{p}^{0.56} \times T_{b}^{0.46}
$$

关于放电通道半径, IKAI 等 ${ }^{[13]}$ 根据大量的试验 结果提出了放电通道半径的经验公式

$$
R_{p}=(2.04 e-3) T_{b}^{0.44} I_{p}^{0.43}
$$

\section{2 初始和边界条件}

脉冲放电温度场仿真模型的初始和边界条件如 图 4 所示。

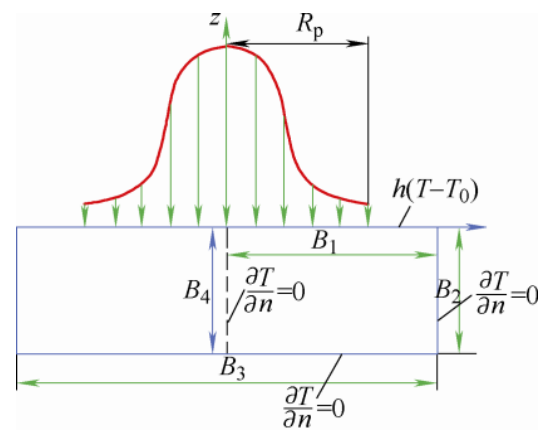

图 4 模型边界条件

在电火花线切割加工过程, $\mathrm{B} 1$ 边界的放电通道 内部为热传导, 放电通道外部为热对流

$$
K \frac{\partial T}{\partial z}=\left\{\begin{array}{l}
q(r) \quad r \leqslant R_{p} \\
h_{c}\left(T-T_{0}\right) \quad r>R_{p}
\end{array}\right.
$$

式中, $h_{c}$ 是介质流体的传热系数; $T_{0}$ 是初始温度。

$B_{2} 、 B_{3} 、 B_{4}$ 边界为热绝缘

$$
\frac{\partial T}{\partial n}=0
$$

\section{3 网格模型的建立}

由于电火花线切割加工过程放电能量集中、热 影响区小，为了提高计算效率，建立了 $80 \mu \mathrm{m} \times 60$ $\mu \mathrm{m} \times 30 \mu \mathrm{m}$ 大小的有限元模型, 区域网格划分为
SOLID 70 六面体网格单元, 边长 $0.5 \mu \mathrm{m}$, 网格模型 如图 5 所示。

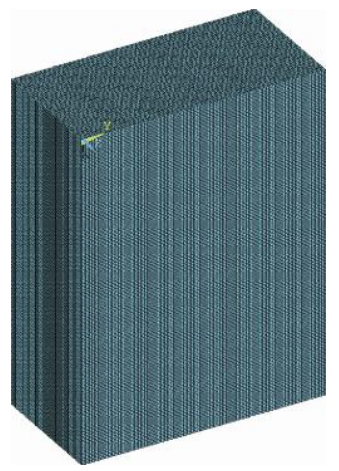

图 5 网格模型

\section{4 材料的热物理性能参数}

$\mathrm{V} 40$ 铇钢密度 $14.5 \mathrm{~g} / \mathrm{cm}^{3}$, 熔点 $2850{ }^{\circ} \mathrm{C}$, 热物 理性能参数如表 1 所示, 在 ANSYS 中输入不同温 度的材料热物性参数值, 系统会自动根据输入的离 散值进行线性插值 ${ }^{[14-15]}$ 。温度超过 $1000{ }^{\circ} \mathrm{C}$ 时, 本 文采用延伸法进行处理。

表 1 V40 钨钢热物性参数表

\begin{tabular}{cccc}
\hline 温度 $T /{ }^{\circ} \mathrm{C}$ & $\begin{array}{c}\text { 导热系数 } \lambda \\
\left(\mathrm{W} \cdot\left(\mathrm{m} \cdot{ }^{\circ} \mathrm{C}\right)^{-1}\right)\end{array}$ & $\begin{array}{c}\text { 比热容 } C / \\
\left(\mathrm{J} \cdot\left(\mathrm{kg} \cdot{ }^{\circ} \mathrm{C}\right)^{-1}\right)\end{array}$ & $\begin{array}{c}\text { 焓 } \mathrm{HI} \\
\left(\times 10^{9} \mathrm{~J} \cdot \mathrm{m}^{-3}\right)\end{array}$ \\
\hline 20 & 75 & 212 & 0 \\
100 & 74 & 240 & 1.17 \\
200 & 71 & 288 & 1.86 \\
300 & 67 & 324 & 2.52 \\
400 & 62 & 360 & 3.47 \\
500 & 58 & 396 & 3.85 \\
600 & 56 & 437 & 4.28 \\
700 & 52 & 472 & 4.61 \\
800 & 50 & 494 & 5.25 \\
900 & 48 & 476 & 5.69 \\
1000 & 47 & 457 & 6.12 \\
\hline
\end{tabular}

\section{3 加载与求解}

考虑到电火花加工过程的复杂性，为了提高计 算效率, 本文对仿真模型做出如下简化, 具体假设 如下所述。

（1）每一次脉冲放电只形成一个放电通道，工 件表面只有最上层的单元参与脉冲放电。

(2) 工件材料均匀，具有各向同性。

（3）传热主要通过热传导和对流换热。

(4) 连续脉冲放电过程的放电通道中心位置沿 电极丝运动方向随机分布。

在电火花线切割连续脉冲随机放电仿真过程 中, 建立存储加工区域的加载单元的随机数组 
Rand, 然后依次选择随机数组存储的加载单元作为 放电通道的中心位置进行加载、求解, 并基于脉冲 放电过程的温度场分布, 引入生死单元技术 “杀死” 超过等效蚀除温度 $T_{e q}$ 的单元, 接着删除载荷、冷 却, 重复循环上述过程直至随机数组 Rand 的单元 被全部调用。图 6 为电火花线切割连续脉冲随机放 电过程的仿真流程图。

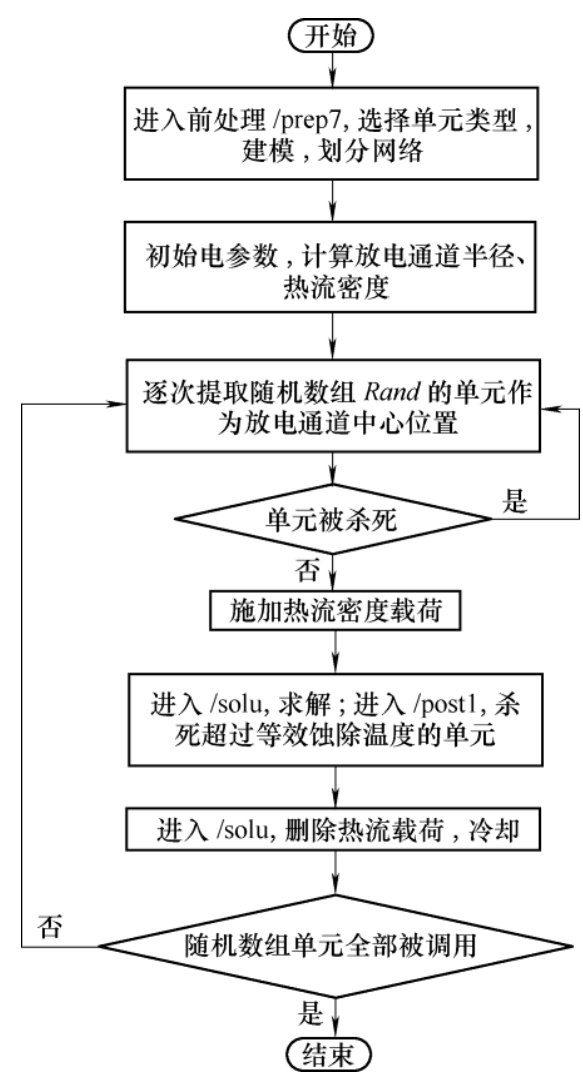

图 6 线切割连续脉冲随机放电仿真流程图

\section{4 仿真结果及分析}

电火花线切割连续脉冲随机放电的仿真过程采 用峰值电流 $2 \mathrm{~A}$ 、脉宽 $4 \mu \mathrm{s}$ 的电参数, 在 $x=(0 \sim 80)$ $\mu \mathrm{m} 、 y=30 \mu \mathrm{m} 、 z=0 \mu \mathrm{m}$ 的 $x$ 方向区域施加连续脉冲, 图 7 是仿真过程中不同时刻工件表面的温度场分 布。基于温度场分布，利用生死单元技术 “杀死” 超过等效蚀除温度 $T_{\mathrm{eq}}$ 的单元, 得到工件表面蚀除 效果如图 8 所示。

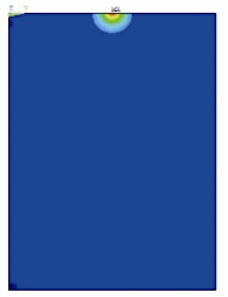

(a) $4 \mu \mathrm{s}$
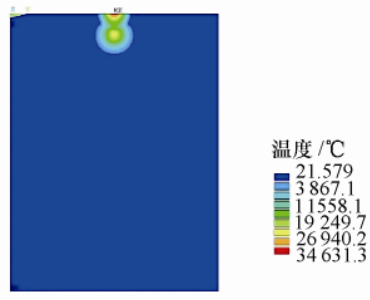

(b) $16 \mu \mathrm{s}$

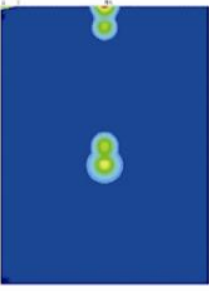

(c) $40 \mu \mathrm{s}$

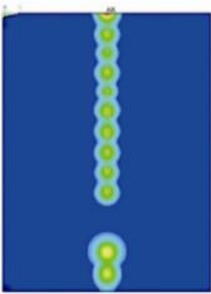

(e) $136 \mu$

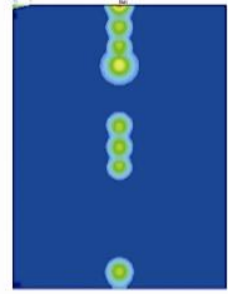

(d) $88 \mu \mathrm{s}$

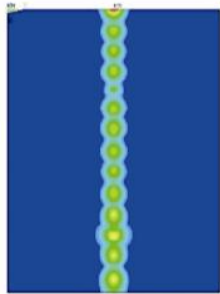

(f) $160 \mu \mathrm{s}$

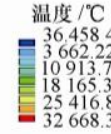

图 7 连续脉冲随机放电过程温度场分布

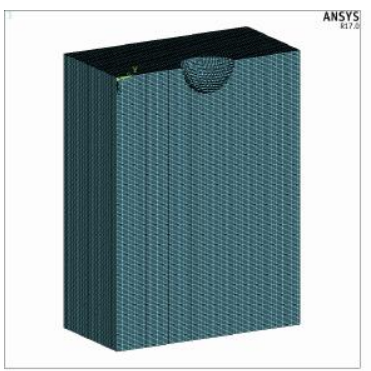

(a) $4 \mu \mathrm{s}$

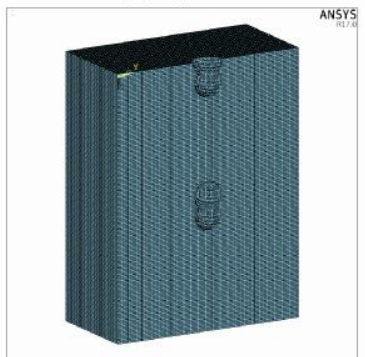

(c) $40 \mu \mathrm{s}$

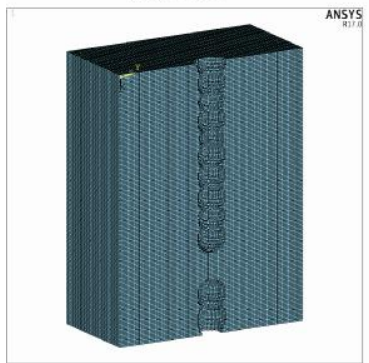

(e) $136 \mu \mathrm{s}$

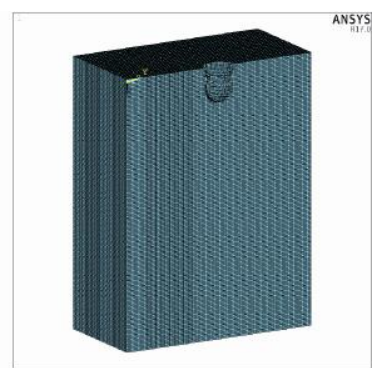

(b) $16 \mu \mathrm{s}$

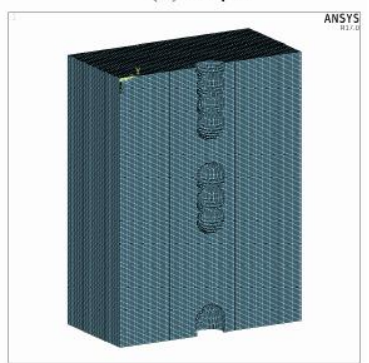

(d) $88 \mu \mathrm{s}$

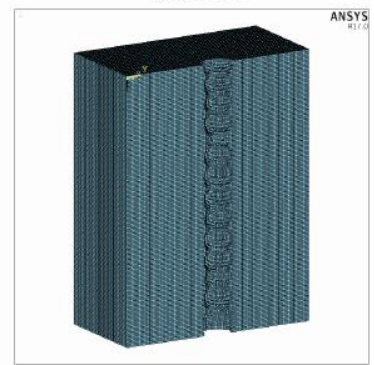

(f) $160 \mu \mathrm{s}$
图 8 连续脉冲随机放电凹坑蚀除过程

从图 7、8 可以看出, 每次脉冲放电结束后, 通 道中心的最高温度、温度场分布和材料去除量并不 一致，出现差异的原因是先前脉冲放电蚀除凹坑和 残留温度场的作用使后续单脉冲放电过程的初始温 度和有效加载面积不完全相同。不同放电脉冲的热 流密度和加载时间在相同的电加工参数的条件下是 固定的, 但有效加载面积和初始温度分布通常不同, 
有效加载面积越大、初始温度越高, 在加载相同的 热流密度和时间时温度场的最高温度越高, 超过等 效蚀除温度的单元越多、脉冲的材料去除量越大。 因此, 以恒定的单脉冲仿真模型分析电火花线切割 的加工过程是不准确的。

图 9 是在共聚焦显微镜下观察的电火花线切割 加工工件的表面形貌, 可以看出工件表面电蚀凹坑 的大小不一, 且电蚀凹坑存在不同程度的重叠, 与 仿真结果较为一致。

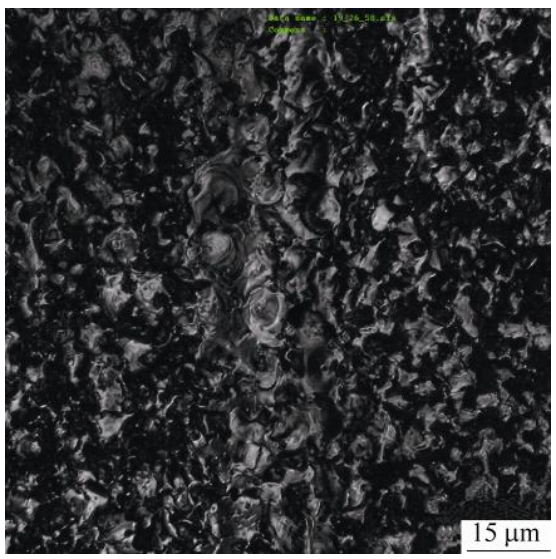

图 9 连续脉冲放电加工表面形貌

为了验证数值模拟的合理性和准确性, 基于仿 真模型, 进行了不同峰值电流的材料去除率试验, 并与实际加工结果进行对比。试验材料选取 $9 \mathrm{~mm}$ 厚 V40 铇钢, 试验加工条件如表 2 所示, 试验结果 如图 10 所示。

表 2 试验加工条件

\begin{tabular}{lc}
\hline 加工参数 & 数值 \\
\hline 开路电压 $/ \mathrm{V}$ & 120 \\
火花维持电压 $/ \mathrm{V}$ & 25 \\
脉宽 $/ \mu \mathrm{s}$ & 4 \\
脉间 $/ \mu \mathrm{s}$ & 20 \\
工作液 & 去离子水 \\
\hline
\end{tabular}

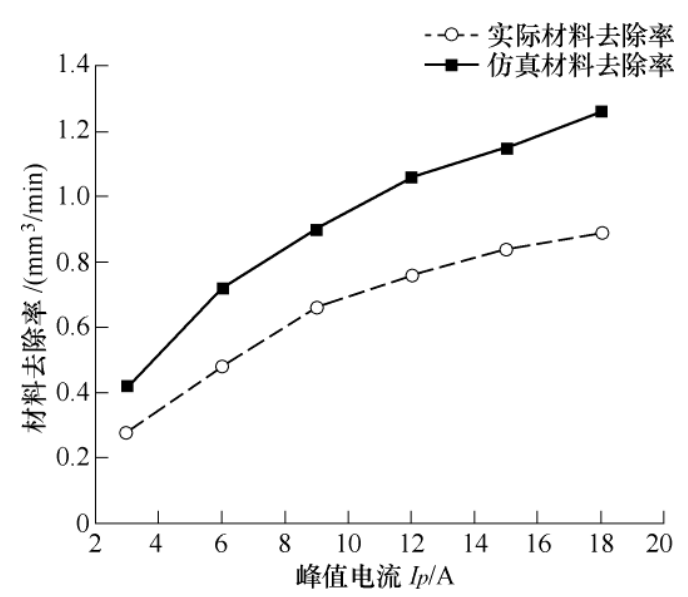

图 10 不同电流条件仿真-实际材料去除率
由图 10 可知, 材料去除率的仿真计算结果随着 电流增大而增大，这是由于电流的增大增加了单脉 冲的能量, 从而升高了工件表面温度场的最高温度, 增大了单脉冲的蚀除体积。在实际加工过程中, 虽 然材料去除率同样随着电流增大而增大, 但数值小 于数值模拟的结果。这是由于在仿真过程中, 脉冲 利用率默认为 $100 \%$, 而实际加工过程中极间存在 开路、短路等现象，同时仿真时未考虑实际加工过 程中电极丝的换向, 需要对仿真模型进行修正。

为了检测电火花线切割加工过程的脉冲利用 率, 本文搭建了脉冲利用率的检测装置, 如图 11 所示。首先在加工过程中利用信号采集器连续采集 极间电压，采集频率设置为 $10 \mathrm{MHz}$ ，每次采集时 长 $3 \mathrm{~s}$, 然后数据传输到计算机端进行统计分析, 图 12 为表 2 试验加工条件下采集的部分极间电压 波形。

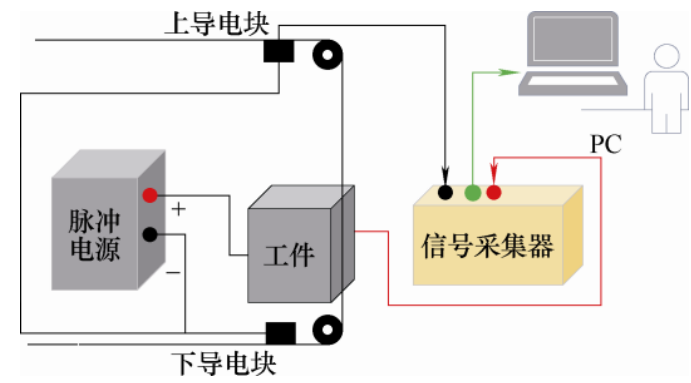

图 11 脉冲利用率检测装置示意图

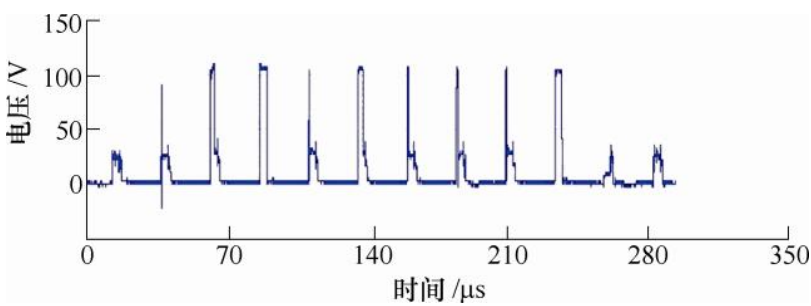

图 12 线切割极间电压波形

在电火花线切割加工过程中, 影响脉冲利用率 的主要因素是伺服参考电压, 因此本文利用脉冲利 用率检测装置统计了不同伺服参考电压下的脉冲 利用率, 如表 3 所示, 其中峰值电流为 $12 \mathrm{~A}$, 脉 宽为 $4 \mu \mathrm{s}$, 脉间为 $20 \mu \mathrm{s}$ 。由表 3 可知, 在现有设 备电火花线切割加工过程中, 脉冲利用率约为 $81 \% \sim 91 \%$ 。加工过程电极丝的换向时间，本文认 为对试验结果的影响比例为 $10 \%$ 。因此, 仿真数据 的修正系数采用 0.75 , 修正后结果如图 13 所示。 可以看出, 修正后材料去除率与实际加工结果之间 误差约为 $10 \%$, 较为吻合, 验证了本文提出的电火 花线切割加工连续脉冲随机放电仿真模型计算结 果的合理性。 
表 3 脉冲利用率试验结果

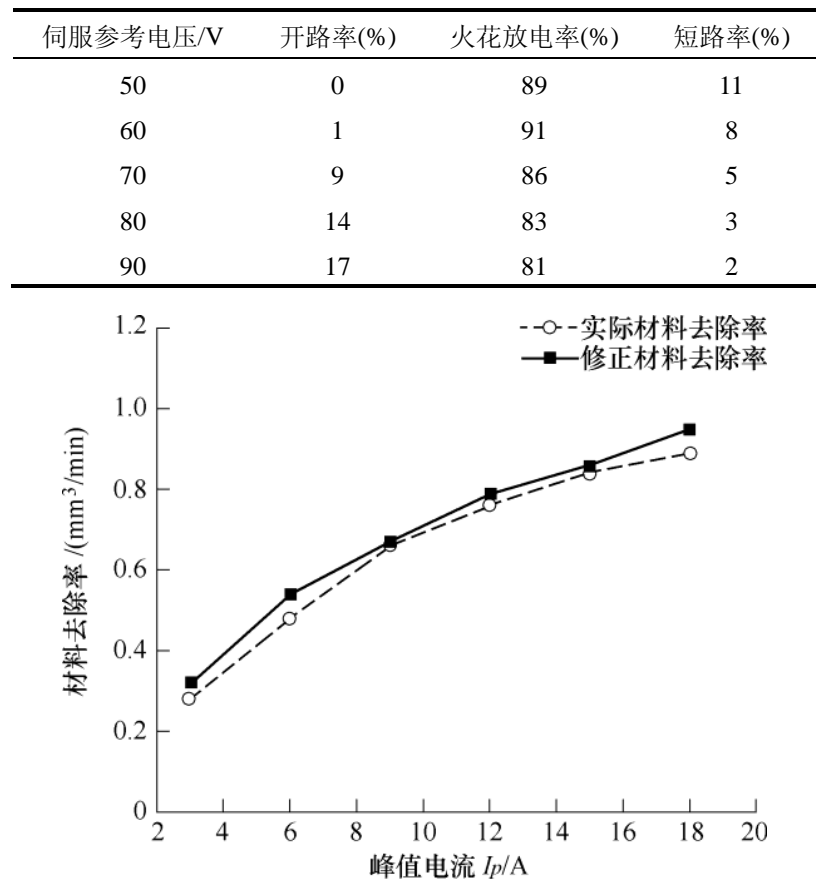

图 13 材料去除率修正结果

\section{5 结论}

（1）通过利用极间电场仿真模型计算极间电场 强度分布, 并分析连续脉冲放电过程的放电点位置 分布, 发现放电位置的分布具有随机性和复杂性, 确定了电火花线切割加工过程中放电位置随机分布 的原则。

（2）基于连续脉冲放电中心位置随机分布的原 则, 建立了 V40 铇钢电火花线切割加工的仿真模型, 仿真分析了连续脉冲放电过程工件表面的温度场分 布, 并引入 “生死单元技术” 得到了材料在加工过 程的表面蚀除情况, 发现连续脉冲放电过程中不同 脉冲的最高温度和放电凹坑体积并不相同, 放电脉 冲之间存在相互作用。

(3) 引入脉冲利用率、电极丝换向时间修正系 数修正材料去除率的仿真结果, 发现与实际加工结 果之间的误差小于 $10 \%$, 验证了电火花线切割加工 连续脉冲随机放电仿真模型的准确性。

\section{参 考 文 献}

[1] 赵万生. 先进电火花加工技术[M]. 北京: 国防工业出 版社, 2003.

ZHAO Wansheng. Advanced electrical discharge machining technology[M]. Beijing: National Defense Industry Press, 2003.

[2] 程国柱, 刘志东, 田宗军, 等. 基于 ANSYS 电火花线 切割加工的温度场分析 $[J]$. 电加工与模具, 2008(6):
24-26.

CHENG Guozhu , LIU Zhidong , TIAN Zongjun, et al. Temperature field analysis based on ANSYS in WEDM[J]. Electromachining \& Mould, 2008(6): 24-26.

[3] 谷萌, 李丽, 王飞飞, 等. 镍基合金 IN718 电火花线 切割单脉冲放电温度场分析 $[\mathrm{J}]$. 机械设计与制造, 2013(9): 151-154.

GU Meng, LI Li, WANG Feifei, et al. The temperature field analysis of nickel-base alloy IN718 in WEDM single pulse discharge[J]. Machinery Design \& Manufacture, 2013(9): 151-154.

[4] 张云卿. 基于 ANSYS 的气中线切割加工温度场及蚀除 量仿真[D]. 哈尔滨: 哈尔滨理工大学, 2015.

Zhang Yunqing. Simulation of temperature field and erosion amount of WEDM in gas based on ANSYS [D]. Harbin: Harbin University of Science and Technology, 2015.

[5] KURIACHEN B, VARGHESE A, SOMASHEKHAR K $\mathrm{P}$, et al. Three-dimensional numerical simulation of microelectric discharge machining of Ti-6Al-4V $[\mathrm{J}]$. The International Journal of Advanced Manufacturing Technology, 2015, 79(1-4): 147-160.

[6] TALLA G, SAHOO D K, GANGOPADHYAY S, et al. Modeling and multi-objective optimization of powder mixed electric discharge machining process of aluminum/alumina metal matrix composite[J]. Engineering Science and Technology, an International Journal, 2015, 18(3): 369-373.

[7] SHANTANU S S, PANDE S. Development of a thermo-physical model for multi-spark wire EDM process[J]. Procedia Manufacturing, 2016， 5: 205-219.

[8] SOMASHEKHAR K P, PANDA S, MATHEW J, et al. Numerical simulation of micro-EDM model with multi-spark [J]. The International Journal of Advanced Manufacturing Technology, 2015, 76(1-4): 83-90.

[9] LIM H J, DOMBLESKY J P, KIM N, et al. FE simulation of material removal and surface properties for EDM during single and multiple spark occurrences $[\mathrm{C}] / /$ Tech. Connect World Innovation Conference, 2015.

[10] OKADA A, UNO Y, NAKAZAWA M, et al. Evaluations of spark distribution and wire vibration in wire EDM by high-speed observation[J]. CIRP Annals-Manufacturing Technology, 2010, 59(1): 231-234.

[11] JOSHI S N, PANDE S S. Thermo-physical modeling of die-sinking EDM process[J]. Journal of Manufacturing Processes, 2010, 12(1): 45-56.

[12] SINGH H. Experimental study of distribution of energy during EDM process for utilization in thermal models[J]. International Journal of Heat and Mass Transfer, 2012, 
55(19): 5053-5064.

[13] IKAI T, HASHIGUSHI K. Heat input for crater formation in EDM[C] // Proceedings of International Symposium for Electro Machining-ISEM XI, EPFL, Lausanne , Switzerland. 1995: 163-170.

[14] 张建华, 孟艳华, 刘承帅, 等. 超声振动辅助气中放电 加工温度场模拟 [J]. 工具技术，2004，38(9): 69-71.

ZHANG Jianhua, MENG Yanhua, LIU Chengshuai, et al.

Simulation of temperature field of ultrasonic vibration aided electrical discharge machining in gas [J]. Tool Engineering, 2004, 38(9): 69-71.
[15] 谭真, 郭广文. 工程合金热物性[M]. 北京：冶金工业 出版社, 1994.

TAN Zhen, GUO Guangwen. Thermophisical properties of engineering alloy [M]. Beijing: Metallurgical Industry Press, 1994.

作者简介: 白宇飞, 男, 1993 年出生。主要研究方向为电火花加工技术。 E-mail: baiyf_hit@163.com 王振龙(通信作者), 男, 1963 年出生, 博士, 教授, 博士研究生导师。 主要研究方向为特种加工技术、微细加工技术、机电控制技术、加工过 程的智能化技术。

E-mail: wangzl@hit.edu.cn 